\title{
EFFECT OF CARRIER TYPES ON THE PHYSICOCHEMICAL AND DISSOLUTION CHARACTERISTICS OF OFLOXACIN SOLID DISPERSION
}

\author{
Okonogi $S^{1 *}$, Sirithunyalung $\mathrm{J}^{1}$, Sirithunyalig $\mathrm{B}^{1}$, Wolschann $\mathrm{P}^{2}$ and Viernstein $\mathrm{H}^{3}$ \\ ${ }^{1}$ Faculty of Pharmacy, Chiang Mai University, Chiang Mai, 50200, Thailand. \\ ${ }^{2}$ Institute of Theoretical Chemistry and Molecular Biology, University of Vienna, Währinger Straße \\ 17, A-1090 Vienna, Austria \\ ${ }^{3}$ Institute of Pharmaceutical Technology and Biopharmaceutics, University of Vienna, Althanstrasse \\ 14, A-1090 Vienna, Austria.
}

\begin{abstract}
Solid dispersions of ofloxacin (OFX) and of a number of carriers including chitosan and the water soluble polymers polyethylene glycol (PEG) 4000, PEG 20000, and polyvinylpyrrolidone K90 were prepared by solvent evaporation method in order to increase the dissolution of the drug. The solid dispersions were subjected to X-ray diffraction, DSC, and dissolution to characterize their physicochemical and dissolution properties. The results demonstrated a decrease in drug crystallinity at higher amounts of carrier. Dissolution studies indicated that the dissolution rate of OFX was markedly increased in these solid dispersion systems compared with the pure drug. The results also showed that the increase in dissolution rate was higher when the weight fraction of carriers increased. An influence of molecular weight of PEG on OFX dissolution could also be observed. In solid dispersion with 1:9 ratio drug to carrier, PEG 4000 gave highest drug dissolution rate, whereas in $1: 1$ ratio, chitosan seems to be the best carrier for drug release. It was concluded that chitosan might be the carrier of choice for dissolution enhancement in solid dispersions with high content of drug.
\end{abstract}

Keywords : ofloxacin, chitosan, solid dispersion, dissolution, PEG, PVP

\section{INTRODUCTION}

Ofloxacin (OFX) is a potent antibacterial drug, which belongs to the class of fluoroquinolone derivatives (1). A major problem of OFX as well as other compounds of this class is the poor aqueous solubility (2). Therefore, the improvement of OFX dissolution is an important task for enhancing its bioavailability and therapeutic efficiency.

In modern pharmaceutical technology, solid dispersion is one of several strategies to improve the dissolution properties and bioavailability of poorly water soluble drugs using hydrophilic carriers as dispersing agents (3). Among the numerous substances examined for their carrier properties, the commonly used are urea (4) and water soluble polymers (5) such as polyethylene glycol (6) and polyvinylpyrrolidone (7).

Recently, there has been a considerable increase in interest in the utilization of chitosan (CTS), a cationic natural biopolymer produced from deacetylation of chitin, as an excipient in the pharmaceutical industry, because of its abundant advantages; e.g. it is inexpensive, non-toxic, and biodegradable (8). Moreover, CTS exhibits good biocompatibility and safety after parenteral 
administration (9) and has been approved as a food ingredient, a fact that suggests the acceptability of this new excipient for oral drug administration (10-11). From a technological point of view, CTS has been demonstrated to be a good vehicle for enhancing the dissolution properties and bioavailability of a number of poorly water soluble drugs (12-17).

In a previous paper (18), we applied the solid dispersion method using urea and mannitol as carriers to improve the OFX dissolution and reported that the dissolution rates of OFX from OFXurea solid dispersions were significantly higher than that from OFX-mannitol solid dispersions.

To clarify this aspect, a solid dispersion of OFX with a number of carriers were prepared and characterized in the present study. As PEG and PVP were the most commonly used hydrophilic polymers that enhance the dissolution rates of many drugs and taking in account all advantages of chitosan mentioned above, these types of materials were used as carriers in this experiment. Moreover, the effect of molecular weight of PEG was also investigated. Powder X-ray diffractometry and differential scanning calorimetry were used to explain the results.

\section{EXPERIMENTAL}

\section{Materials}

Ofloxacin (OFX) was obtained from Daiichi Seiyaku Co. Ltd (Japan). Chitosan (CTS) with a degree of deacetylation of $85 \%$ was obtained from Ta Ming Enterprises Co. Ltd. (Thailand). The average particle size of CTS quoted by the manufacturer was 80 mesh. Polyethylene glycol (PEG) with different molecular weights (PEG 4000 and PEG 20000) and polyvinylpyrrolidone (PVP) K90 were purchased from Fluka Chemie AG. All other chemicals were of analytical grade.

\section{Preparation of OFX solid dispersions}

The solid dispersions of OFX and different carriers were prepared at weight ratios of 1:1, 3:7, and 1:9 (drug to carrier) by solvent evaporation method using chloroform as solvent. OFX was dissolved ( $1 \mathrm{~g}$ OFX / $50 \mathrm{ml}$ of chloroform) before the carrier was added to the solution. This material containing OFX and water-soluble polymers as well as the colloidal dispersion of CTS was evaporated at $40^{\circ} \mathrm{C}$. After complete evaporation, the solid mass was further dried in a vacuo at room temperature for $12 \mathrm{~h}$. The dried solid mass was then pulverized and sieved and the solid products of 60-200 mesh were used in this experiment.

\section{Powder X-ray diffractometry (PXRD)}

The crystalline characteristics of OFX-CTS solid dispersions were examined by means of a Kristalloflex Siemens D500. The X-ray source was $\mathrm{Cu}-\mathrm{K} \alpha$ with a $\mathrm{Ni}$ filter $(20 \mathrm{kV}, 10 \mathrm{~mA})$ and the scanning speed was $5^{\circ} / \mathrm{min}$.

The OFX-PEG and OFX-PVP solid dispersions were subjected to a Rigaku Denki 2027 diffractometer. The $\mathrm{X}$-ray source was $\mathrm{Cu}-\mathrm{K} \alpha$ with a $\mathrm{Ni}$ filter $(30 \mathrm{kV}, 5 \mathrm{~mA})$ and the scanning speed was $4^{\circ} / \mathrm{min}$. 


\section{Differential scanning calorimetry (DSC)}

A 2-3 mg sample was placed in an aluminium pan. A Perkin-Elmer DSC 7 and a DuPont 9900 thermal analyzer were used for the characterization of solid dispersions consisting of OFXCTS OFX-PEG and OFX-PVP, respectively. The measurements were carried out under a nitrogen gas flow of $60 \mathrm{ml} / \mathrm{min}$ at a heating rate of $10^{\circ} \mathrm{C} / \mathrm{min}$.

\section{Dissolution studies}

Dissolution characteristics of OFX and OFX solid dispersions were investigated at $37^{\circ} \mathrm{C}$ using a modified USP XXV paddle method with a rotating speed of $100 \mathrm{rpm}$. A powder sample containing $100 \mathrm{mg}$ OFX was compressed at $2 \mathrm{t} / \mathrm{cm}^{2}$ to a flat tablet $(10 \mathrm{~mm}$ diameter). The tablet was then fixed at the bottom of the dissolution vessel containing $1000 \mathrm{ml}$ of distilled water as test medium. The side of the tablet was sealed by paraffin to allow only the top surface to contact the medium throughout the dissolution experiment. A $5 \mathrm{ml}$ portion of the solution was taken out periodically, and the same amount of distilled water at the same temperature was replaced. The amount of OFX was determined spectrophotometrically.

\section{RESULTS AND DISCUSSION}

\section{Crystalline characteristics of OFX}

The PXRD patterns, over the $2 \theta$ range from 10 to $40^{\circ}$, of OFX solid dispersions with CTS, PEG 4000, PEG 20000, and PVP are compared with OFX and each of the carriers (Fig. 1). OFX showed numerous distinctive peaks at $2 \theta$ of $10.8,13.7,15.7,20.3$, and $21.8^{\circ}$, indicating a high crystallinity. PEG 4000 and PEG 20000 also exhibited some crystallinity, showing peaks of high intensity at $13.5,19.1,23.2$, and $27.3^{\circ}$ for PEG 4000 and at 19.3 and $23.5^{\circ}$ for PEG 20000 and other peaks of low intensity. PVP depicted halo pattern indicating amorphous form and CTS exhibited a single broad peak of low intensity at $19.9^{\circ}$ (amorphous nature).

No peak indicating crystallinity of OFX was detected from the PXRD patterns of solid dispersions at a ratio of 1:9. This result suggested that on heating in DSC, OFX progressively dissolved in PEG and PVP and dissolved completely below the melting temperature of crystalline OFX. In solid dispersions with higher drug content, e.g. at ratios of 3:7 and 1:1, the PXRD patterns exhibited the identical peaks of OFX at $2 \theta$ of 10.80 and $15.7^{\circ}$, but of low intensity. This reveals that some OFX still existed in the crystalline state in the solid dispersions.

\section{Thermal behavior of OFX}

The DSC thermograms over a temperature range from 50 to $300^{\circ} \mathrm{C}$ of OFX solid dispersions with CTS, PEG 4000, PEG 20000, and PVP were compared to those OFX and each of the carriers (Fig. 2). OFX showed a sharp melting endotherm at $278^{\circ}$ C. PEG 4000 and PEG 20000 exhibited a single sharp endothermic peak at 56.8 and $62.8^{\circ} \mathrm{C}$, respectively. CTS showed no endothermic peak whereas PVP depicted a broad endothermic peak corresponding to water dehydration at $148.5^{\circ} \mathrm{C}$. In the thermograms of OFX-CTS solid dispersions as shown in Fig 2 (A), an endothermic sharp peak at $278^{\circ} \mathrm{C}$ was observed in all samples, but lower in intensity as the content of drug decreased. This indicated the melting process of OFX crystallites existing in the systems. 
(A)

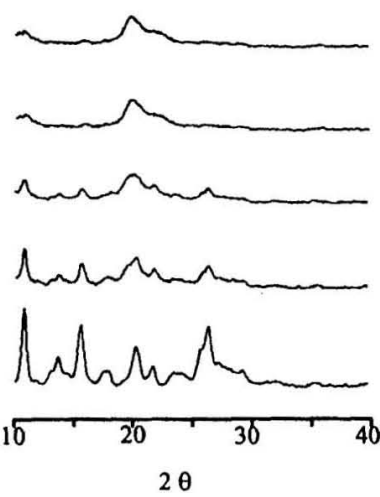

(C)

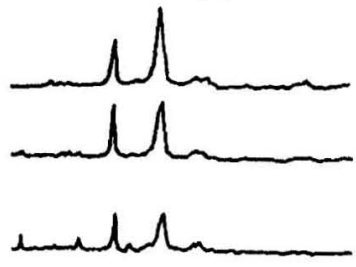

(a)

(b)

(c)

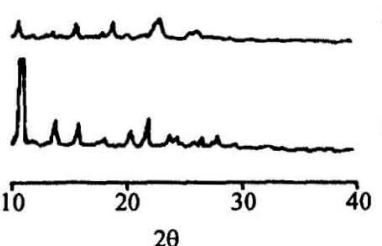

(B)
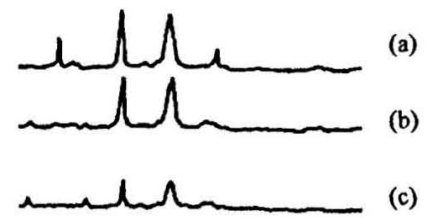

(d)

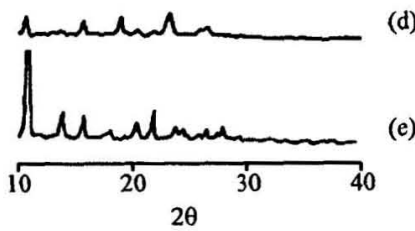

(D)

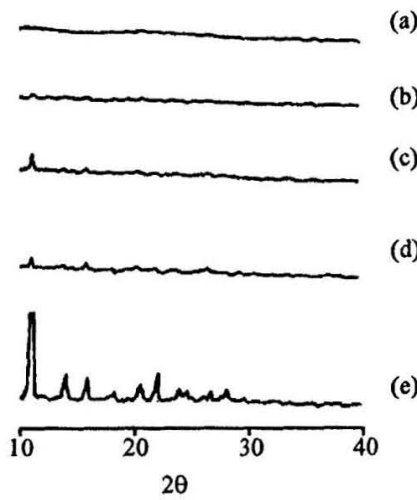

Figure 1 Powder X-ray patterns of (a) carrier, (b) 1:9 solid dispersion, (c) 3:7 solid dispersion, (d) 1:1 solid dispersion, and (e) OFX; (A) CTS, (B) PEG 4000, (C) PEG 20000, and (D) PVP. 
(A)

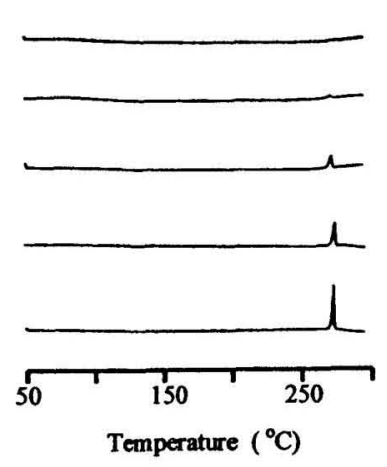

(C)

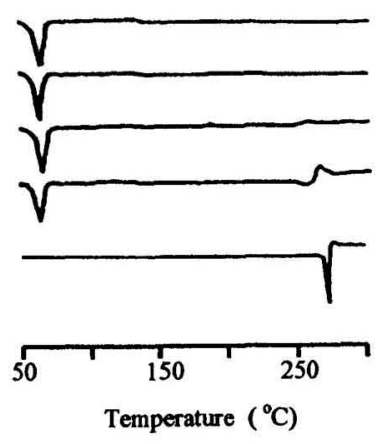

(B)

(a)

(b)

(c)

(e)

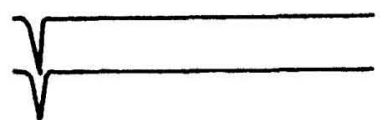

(a)

(c)

(e)

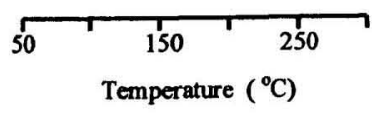

(D)

(a)

(b)

(c)

(d)

(e)

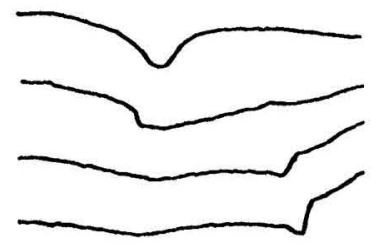

(a)

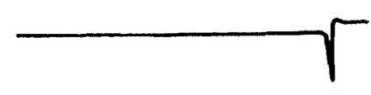

(e)

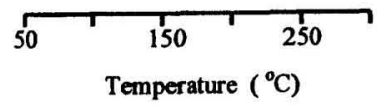

Figure 2 DSC thermograms of (a) carrier, (b) 1:9 solid dispersion, (c) 3:7 solid dispersion,

(d) 1:1 solid dispersion, and (e) OFX; (A) CTS, (B) PEG 4000, (C) PEG 20000, and

(D) PVP.

In the thermograms of OFX-PEG 4000, OFX-PEG 20000, and OFX-PVP solid dispersions as shown in Fig. 2 (B-D), no endothermic peak of OFX was observed when the ratio of drug to carrier is 1:9. This result suggested that on heating in DSC, OFX progressively dissolved in PEG and PVP and dissolved completely below the melting temperature of crystalline OFX. When the content of 
drug was high the broad endothermic peak appeared but much lower in intensity and shifted to the temperature lower than the melting point of OFX whereas the melting point of PEG or PVP was constant. This result demonstrated that OFX formed monotectic mixtures with both PEG and PVP. Other solid dispersions have been found to behave similarly e.g. triamterene, which formed monotectic mixtures with PEG and Gelucire $44 / 14(19,20)$ and cinnarizine, which formed monotectic mixtures with Gelucire 53/10 (21). Lloyd (22) and Okonogi (23) have previously suggested that the drug may dissolve in the molten polymer over the range of temperature; hence the melting endotherm of OFX in those solid dispersions broadened to such an extent that they could not be distinguished from the baseline. The data presented here largely support this hypothesis.

\section{Dissolution studies}

The dissolution profiles of OFX from the solid dispersions are shown in Fig. 3. In all cases, the dissolution of OFX was improved. Solid dispersions with a drug to carrier ratio of 1:9 ratio exhibited faster dissolution compared with those having a drug to carrier ratio of $1: 1$. In solid dispersions (1:9 ratio), the order for the carriers to enhance drug release within the first $30 \mathrm{~min}$ (Tab. 1) was PEG $4000>$ PEG $20000>$ CTS $>$ PVP.

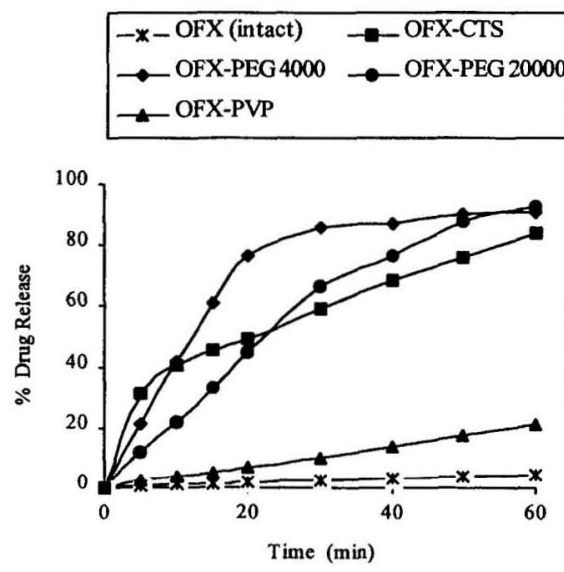

(A)

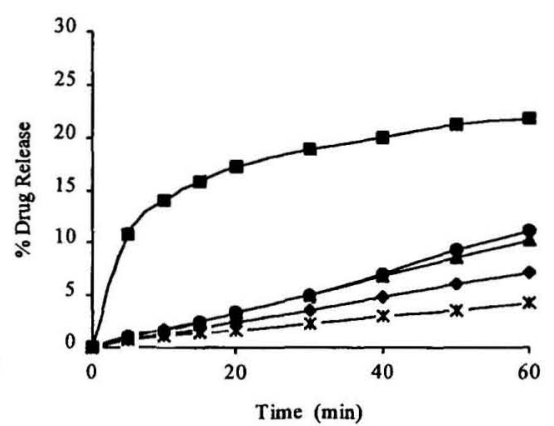

(B)

Figure 3 Dissolution profiles of OFX from 1:9 (A) and 1:1 (B) solid dispersions 
Table 1 Dissolution of OFX and release from solid dispersions within $30 \mathrm{~min}$.

\begin{tabular}{|c|c|c|}
\hline \multirow{2}{*}{ drug / solid dispersions } & \multicolumn{2}{|c|}{ drug release (\%) } \\
\cline { 2 - 3 } & $\mathbf{1 : 9}$ ratio & $\mathbf{1}: \mathbf{1}$ ratio \\
\hline OFX-CTS & 58.7 & 18.9 \\
\hline OFX-PEG 4000 & 85.9 & 3.6 \\
\hline OFX-PEG 20000 & 66.4 & 5.0 \\
\hline OFX-PVP & 13.5 & 5.1 \\
\hline OFX & 2.3 & 2.3 \\
\hline
\end{tabular}

OFX-PEG 4000 gave the most improvement at a 1:9 ratio. This was due to the improvement of the wetting of the hydrophobic OFX crystals. When the ratio of drug to carrier was increased to $1: 1$, the order for carriers to enhance drug release within the first $30 \mathrm{~min}$ was significantly changed to CTS > PVP > PEG $20000>$ PEG 4000. At this ratio, OFX-CTS exhibited the highest burst release within the first $30 \mathrm{~min}$. Other carriers were found to behave similarly with respect to the drug. In the process of solid dispersion preparation, CTS was insoluble in chloroform and gave a colloidal dispersion. During solvent evaporation when the drug was precipitated, OFX might deposit as crystallites on the surface of insoluble microparticulated CTS as surface solid dispersion. This caused the increase in surface area of OFX in contact with dissolution medium. CTS was not dissolved in water, therefore, it did not cause a high viscosity of the dissolution medium whereas the water soluble polymer gave a high viscosity. Moreover, CTS showed fast disintegrating action. Hence, formulations with CTS showed a fast liberation of the drug as well as the highest concentrations in the test medium.

\section{ACKNOWLEDGEMENT}

This study was supported by the National Research Council of Thailand (NRCT). The authors would like to thank Daiichi Seiyaku Co. Ltd. (Japan) and Ta Ming Enterprises Co. Ltd. (Thailand) for their kind supply of OFX and CTS respectively.

\section{REFERENCES}

1. Hooper DC and Wolfson JS. 1991. Fluoroquinilone antibacterial agents. The New Eng. J. Med. 324, 384-394.

2. Ross DL and Riley CM. 1990. Aqueous solubilities of some variously substituted quinolone antibacterials. Int.J.Pharm. 63, 237-250.

3. Ford JL. 1986. The current status of solid dispersions. Pharm.Acta.Helv. 61, 69-88.

4. Okonogi S, Yonemochi E, Oguchi T, Puttipipatkhachorn S, and Yamamoto K. 1997. Enhanced dissolution of ursodeoxycholic acid from the solid dispersion. Drug Dev. Ind. Pharm. 23, 1115-1121.

5. Sugimoto M, Okagaki T, Narisawa S. Koida Y, and Nakajima K. 1998. Improvement of dissolution characteristics and bioavailability of poorly water-soluble drugs by novel cogrinding method using water-soluble polymer. Int. J. Pharm. 160, 11-19. 
6. Craig DQM. 1990. Polyethylene glycols and drug release. Drug Dev. Ind. Pharm. 16, 25012526.

7. Sugimoto I, Kuchiki A, Nakagawa H, Tohgo K, Kondo S, Iwane I, and Takahashi K. 1980. Dissolution and absorption of nifedipine from nifedipine-polyvinylpyrrolidone coprecipitates. Drug Dev. Ind. Pharm. 6, 137-160.

8. Lehr CM, Bouwstra JA, Schacht EH, and Junginger HE. 1992. In vitro evaluation of mucoadhesive properties of chitosan and some other natural polymers. Int. J. Pharm. 78, 4348.

9. Hirano S, Seino H, Akiyama I, and Nonaka I. 1990. Chitosan - a biocompatable for oral and intravenous administrations. In: Gebelein CG, Dunn RL (Eds.), Progress in Biomedical Polymers. Plenum Press, New York, pp. 283-289.

10. Weiner ML. 1993. An overview of the regulatory status and of the safety of chitin and chitosan as food and pharmaceutical ingredients. In: Brine CJ, Sanford PA, and Zizakis JP (Eds.), Advances in chitin and chitosan. Elsevier, London, pp 663-672.

11. Illum L. 1998. Chitosan and its use as a pharmaceutical excipient. Pharm. Res. 15, 1326-1331.

12. Sawayanagi Y, Nambu N, and Nagai T. 1982. Enhancement of issolution properties of griseofulvin from ground mixtures with chitin or chitosan. Chem. Pharm. Bull. 30, 44644467.

13. Sawayanagi Y, Nambu N, and Nagai T. 1983. Dissolution properties and bioavailability of phenytoin from ground mixtures with chitin and chitosan. Chem. Pharm. Bull. 31, 2064-2068.

14. Shiraishi S, Arahira M, Imai T, and Otagiri M. 1990. Enhancement of dissolution rates of several drugs by low-molwcular weight chitosan and alginate. Chem. Pharm. Bull. 38, 185189.

15. Acarturk F, Sencan A, and Celebi N. 1993. Enhancement of the dissolution of spironolactone with chitosan and low-molecular weight gelatin. STP. Pharma. Sci. 3, 369-373.

16. Genta I, Pavanetto F, Conti B, Giunchedi P, and Conte U. 1995. Improvement of dexamethasone dissolution rate from spray-dried chitosan microspheres. STP. Pharma. Sci. 5, 202-207.

17. Portero A, Remunan-Lopez C, and Vila-Jato JL. 1998. Effect of chitosan and chitosan glutamate enhancing the dissolution properties of the poorly water soluble drug nifedipine. Int. J. Pharm. 175, 75-84.

18. Okonogi S, Oguchi T, Yonemochi E, Puttipipatkhachorn S, and Yamamoto K. 1997. Improved dissolution of ofloxacin via solid dispersion. Int. J. Pharm. 156, 175-180.

19. Craig DQM and Newton JM. 1991. Characterization of polyethylene glycol solid dispersions using differential scanning calorimetry and solution calorimetry. Int. J. Pharm. 76, 17-24.

20. Dordunoo SK, Ford JL, and Rubinstein MH. 1991. Preformulation studies on solid dispersions containing triamterene or temazepam in polyethylene glycols or Gelucire 44/14 for liquid filling of hard capsules. Drug Dev. Ind. Pharm. 17, 1685-1713.

21. Gines JM, Veiga MD, Arias MJ, and Rabasco AM. 1995. Elaboration and thermal study of interactions between cinnarizine and Gelucire 53/10 physical mixtures and solid dispersions. Int. J. Pharm. 126, 287-291.

22. Lloyd GR, Craig DQM, and Smith A. 1997. An investigation into the melting behavior of binary mixes and solid dispersions of paracetamol and PEG 4000. J. Pharm. Sci. 86, 991-996.

23. Okonogi S, Puttipipatkhachorn S, and Yamamoto K. 2001.Thermal behavior of ursodeoxycholic acid in urea: Identification of anomalous peak in the thermal analysis. Drug Dev. Ind. Pharm. 27, 819-823. 